\title{
Peritalar Dislocation: A Case Report and Literature Review
}

\author{
by Dr. Oliver Blocker MRCS ${ }^{1 凶}$, Dr. Jacqui Orme MBBS $^{2}$, Dr. Anirudh Gadgil FRCS (Ortho) ${ }^{3}$
}

\section{The Foot and Ankle Online Journal 2 (11): 3}

A case of closed medial peritalar dislocation is presented. Immediate closed reduction and conservative management of associated fractures followed by early physiotherapy led to an excellent outcome of this potentially devastating injury. A review of the literature on this rare injury is also discussed.

Key Words: Peritalar dislocation, subtalar dislocation.

\section{Case Report}

A seventy four year-old male patient presented with severe pain and the inability to bear weight after sustaining an inversion injury to the right ankle following a three feet fall from a ladder. This was a closed injury without neurovascular compromise. The ankle and rearfoot were deformed. (Fig.1)

Plain radiographs showed the dislocation of the talonavicular and subtalar joints, with the navicular and calcaneus displaced medially. (Figs. 2 and 3) A lateral view also showed an avulsion fracture of the posterior process of the talus. A closed reduction was performed under general anesthesia using image intensifier guidance.

Address correspondence to: West Wales General Hospital (WWGH), Dolgwili Road, Carmarthen, Dyfed, SA31 2AF, WALES.

${ }^{1}$ Dr. Oliver Blocker MRCS - CT1 Trauma and Orthopaedics, WWGH, 3 Glyn Hirnant, Clydach Road, Morriston, Swansea, SA6 6QS, WALES.

2 Dr. Jacqui Orme MBBS - F2 Trauma and Orthopaedics, WWGH , 3 Glyn Hirnant, Clydach Road, Morriston, Swansea, SA6 6QS, WALES.

${ }^{3}$ Dr. Anirudh Gadgil FRCS(Ortho) - Consultant Foot and Ankle Surgeon, WWGH, 3 Glyn Hirnant, Clydach Road, Morriston, Swansea, SA6 6QS, WALES.
A post-operative computed tomography (CT) scan confirmed anatomical reduction of subtalar and talonavicular joints and also demonstrated a vertical posterior process fracture of the talus extending to the subtalar joint as well as a fracture of the anterior process of the calcaneus extending into the calcaneocuboid joint. (Fig. 4) These were in an acceptable anatomical position and no further surgical intervention was undertaken. The patient was treated in a plaster of Paris, below knee, non-weight bearing cast for a period of 6 weeks and was then allowed to weight bear. Physiotherapy in the form of active and passive mobilization of the ankle, rearfoot and midfoot joints began at 6 weeks. Radiographs revealed the fracture had united at three months follow up and the patient was pain free in his daily activities involving weight bearing. (Fig. 5) 


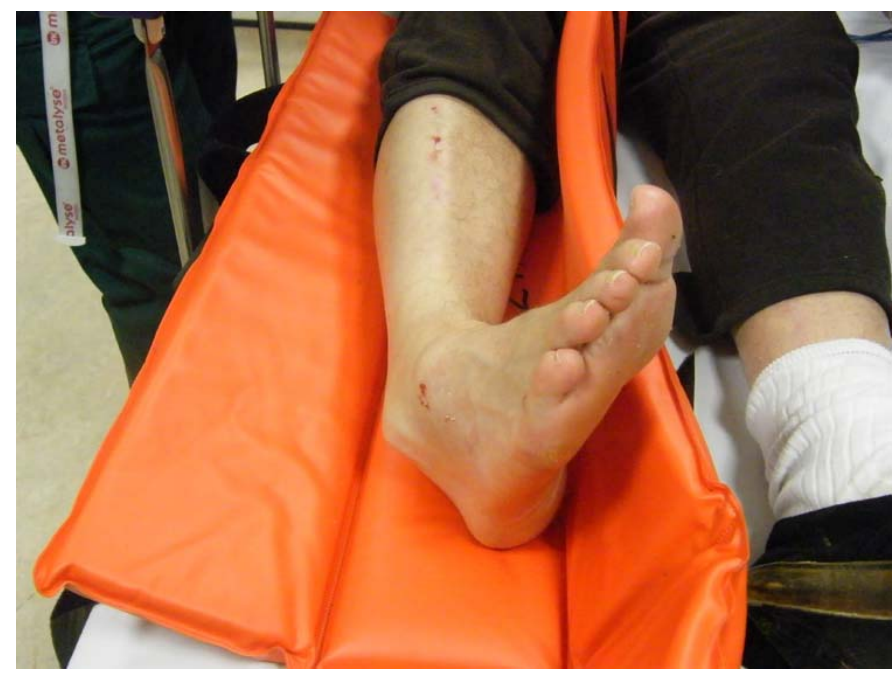

Figure 1 Clinical photograph showing a medially dislocated rearfoot.

\section{Discussion}

Peritalar dislocation refers to the injury involving the simultaneous dislocation of both the talocalcaneal and talonavicular joints without a fracture of the talar neck or tibiotalar joint disruption. ${ }^{1}$ It is rare and considered to be a severe injury as it involves the weight bearing articular surfaces of the subtalar joints. ${ }^{2,3}$

There are two types of normal movements that occur at the talocalcaneal and talonavicular joint. These have been described as 'triplanar' movements. ${ }^{4}$ They are inversion, plantarflexion and adduction as one movement and eversion, dorsiflexion and abduction as the other. This gives the peritalar joint the potential for a variety of dislocations. A medial dislocation is the most common although dislocations can occur laterally, anteriorly and posteriorly. ${ }^{1,5,6,7}$ Peritalar dislocation is usually the result of highenergy trauma or sporting injuries and accounts for $1 \%$ of all dislocations. ${ }^{1,8}$

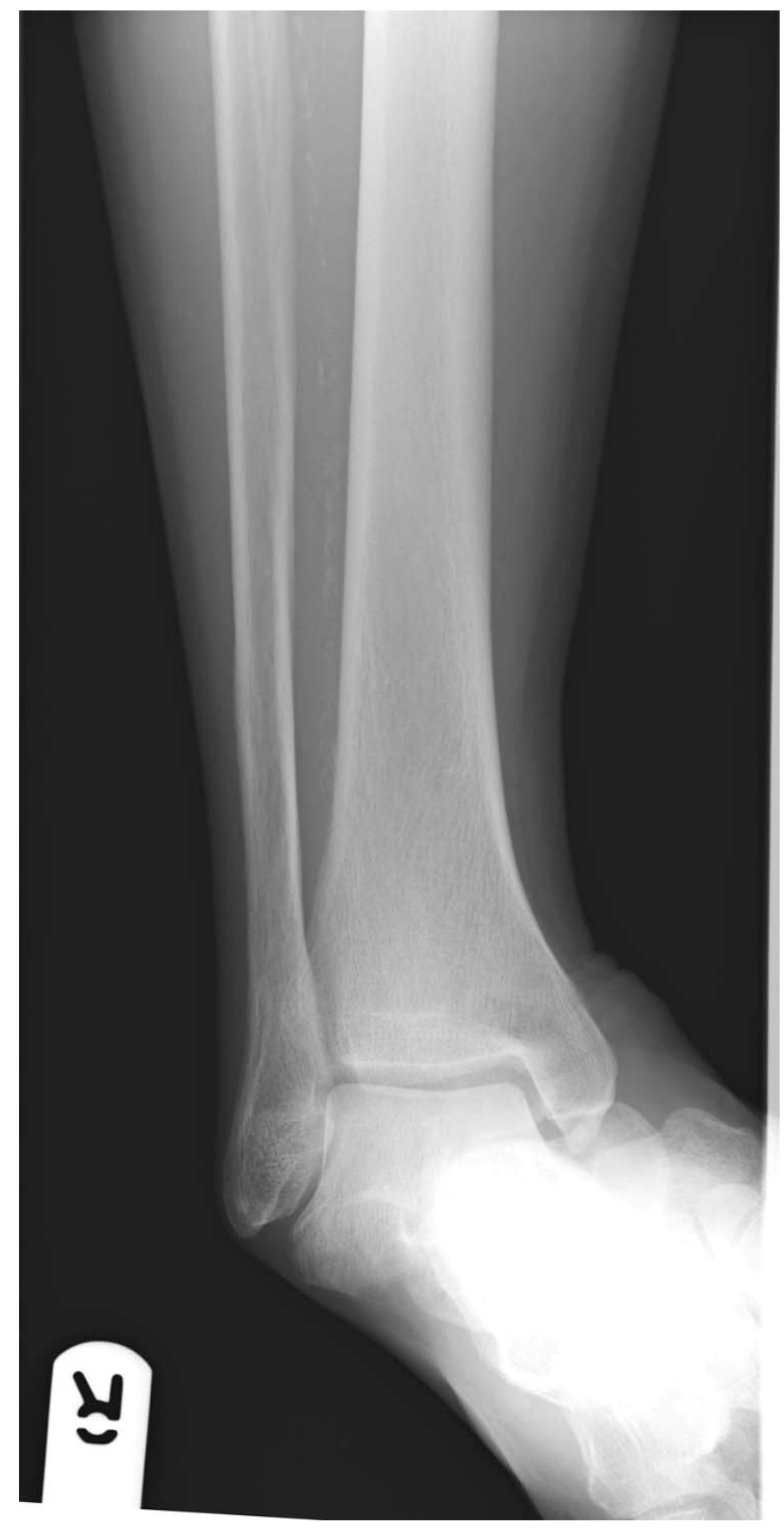

Figure 2 Antero-posterior radiograph demonstrating intact tibio-talar articulation and the disarticulation between the head of the talus and the navicular, with the navicular and calcaneum being displaced medially.

Although open reduction and internal fixation of talar fractures is an option, ${ }^{9}$ the literature suggests that closed reduction is the most common and appropriate method of treatment for medial dislocations. ${ }^{1,3,4,6,10,11}$ 


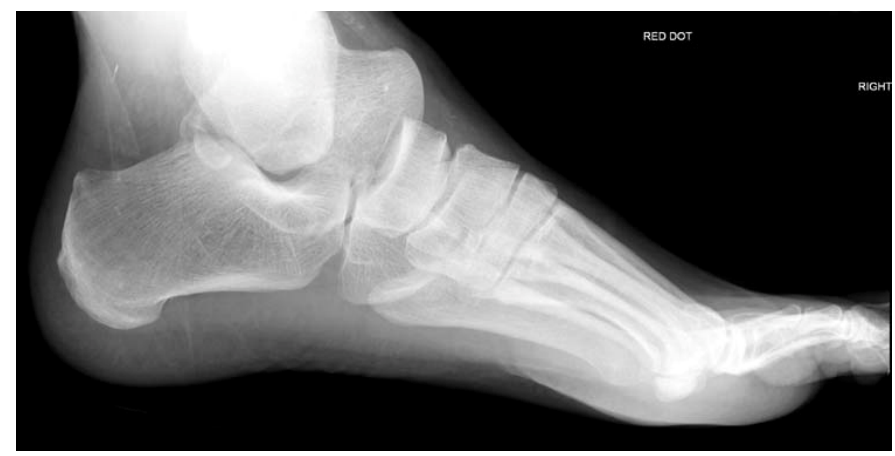

Figure 3 Lateral view radiograph of the ankle and foot. Avulsion fractures of the posterior process of the talus and anterior process of the calcaneum are seen. The dislocated talonavicular joint is seen. Incongruity of the subtalar articulation gives an indication to the subtalar dislocation.

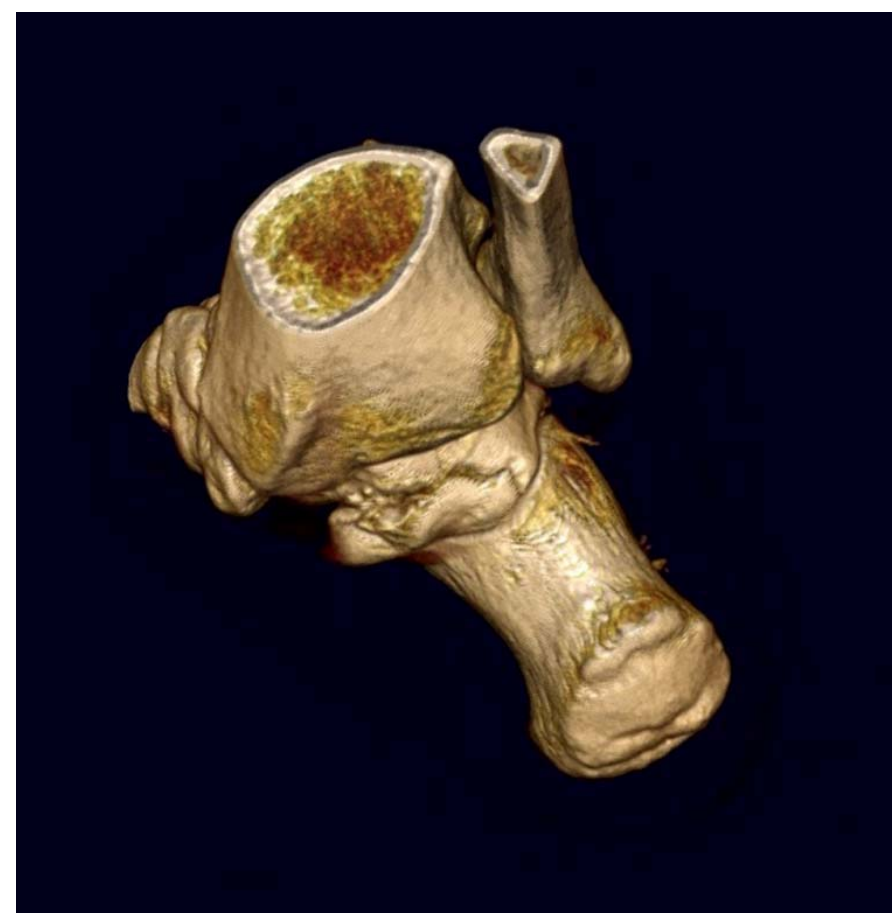

Figure 4 Three dimensional (3D) reconstruction of the post reduction $\mathrm{CT}$ scan shows a vertical fracture of the posterior process of the talus and a well-reduced subtalar joint.

Regardless of the reduction method used, early reduction is the key to ensuring a good prognosis for recovery. 8,10

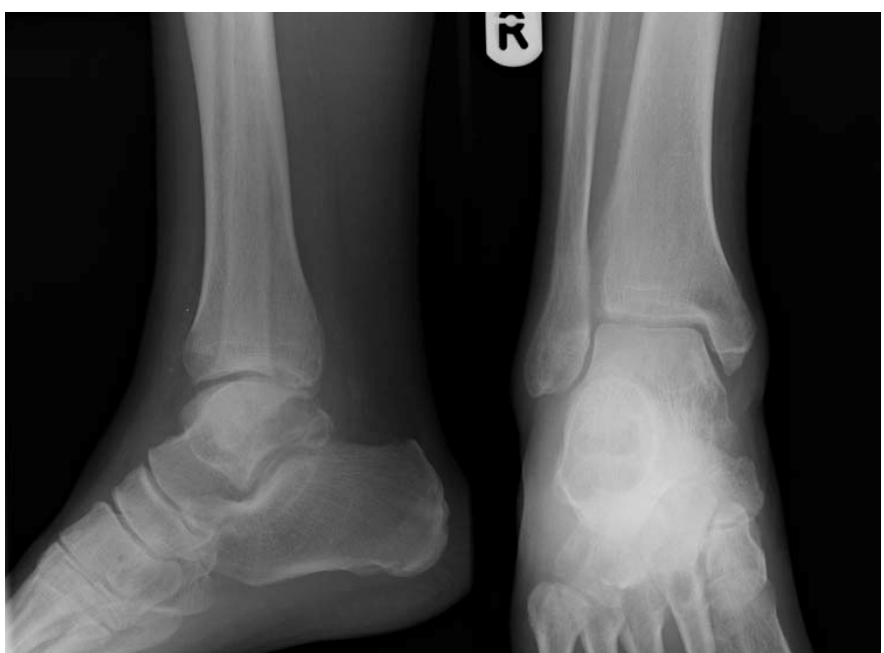

Figure 5 Radiographs taken at the 3-month follow-up show complete bony resolution of the injury with good talonavicular and subtalar joint position.

Peritalar dislocation is a potentially devastating injury $^{12}$ in which the complications range from chronic pain from subtalar arthrosis to talar avascular necrosis. ${ }^{5,9,12}$ Avascular necrosis is rare from simple talar neck fractures as there are multiple vascular anastomoses within the bone itself. The blood supply to the talus is from multiple extra-osseal vessels that surround the bone. ${ }^{13}$ The extensive soft tissue damage that occurs in peritalar dislocation disrupts these vascular arcades. If this is not corrected, avascular necrosis is likely to occur. Therefore early reduction gives the best chance of restoring the blood supply and minimizes the risk of avascular necrosis. Varying disability results from medial dislocations but early reduction and mobilization of the joint is associated with a better prognosis ${ }^{7}$; lateral and open fracture dislocations are associated with poorer outcomes. ${ }^{6}$ Currently, the literature suggests a period of non-weight bearing of 4 weeks with no fracture and 6 weeks with an associated fracture. ${ }^{6}$ Our management included active physiotherapy from the end of the non-weight bearing period until the 3month follow up. We believe this contributed to the patient's rapid recovery. 
The figures included in this report should assist clinicians in the recognition of medial dislocations (Fig. 1), highlight the potential complications such as associated talar fractures (Figs. 3 and 4) and demonstrate the excellent outcomes that can be achieved with early reduction and active rehabilitation (Fig. 5).

In conclusion peritalar dislocations are rare injuries usually sustained by high-energy trauma and have the potential for serious complications such as avascular necrosis of the talus and subtalar arthrosis. However urgent treatment by anatomical reduction followed by early rehabilitation can provide a good outcome.

\section{References}

1. Harris J, Huffman L, Suk M: Lateral peritalar dislocation: a case report. Journal of Foot \& Ankle Surgery 47 (1): 56 - 59. 2008.

2. Simon LC, Schulz AP, Faschingbauer M, Morlock M, Jurgens C: "Basketball Foot" - long-time prognosis after peritalar dislocation. Sportverletzung Sportschaden 22 (1): 31 - 37, 2008. 3. Biswas S, Murphy M. Subtalar dislocation. The Internet Journal of Orthopedic Surgery 2: 3, 2006. Accessed 1 ${ }^{\text {st }}$ March 2008.

4. Ledoux WR, Sangeorzan BJ: Clinical biomechanics of the peritalar joint. Foot \& Ankle Clinics 9 (4): 663 - 683, 2004. 5. Wagner R, Blattert TR, Weckbach A. Talar dislocations. Injury 35 (Suppl 2): SB36 - 45, 2004.

6. Garofalo R, Moretti B, Ortolano V, Cariola P, Solarino G, Wettstein M, Mouhsine E: Peritalar dislocations: a retrospective study of 18 cases. Journal of Foot \& Ankle Surgery 43(3): $166-$ 172, 2004.

7. Merianos P, Papagiannakos K, Hatzis A, Tsafantakis E:

Peritalar dislocation: a follow-up report of 21 cases. Injury 1988 19 (6): 439 - 442, 1988.

8. Maes R, Averous C, Copin G: Lateral peritalar luxation: prognostic evaluation and therapeutic approach. Review of the literature based on a clinical case. Revue Medicale de Bruxelles 24(6): 458 - 463, 2003.

9. Besch L, Drost J, Egbers HJ: Treatment of rare talus dislocation fractures. An analysis of 23 injuries. Unfallchirurg 105 (7): 595 - 601, 2002.

10. Horer DI, Fishman J: The early treatment of peritalar dislocation. International Orthopaedics 7 (4): 263 - 266, 1984. 11. Kumar P, Bajracharya S, Pandey S: Medial peritalar dislocation in a volleyball player. Journal of the Nepal Medical Association 45 (163): 314 - 325, 2006.

12. Marcinko DE, Zenker CC: Peritalar dislocation without fracture. Journal of Foot Surgery 30 (5): 489 - 493, 1991. 13. Mulfinger GL, Trueta J: The blood supply of the talus. Journal of Bone Joint Surgery 52B (1): 160 - 167, 1970.

(C) The Foot and Ankle Online Journal, 2009 